\title{
EXPERIMENTAL CHARACTERISATION OF THE DISPERSION EVOLUTION VERSUS STRETCHING IN A LARGE STROKE OPTICAL FIBRE DELAY LINE
}

\author{
SIMOHAMED.L.M", F.REYNAUD.F".
}

\begin{abstract}
:
In the frame of a large stroke optical fibre delay line implementation, we report experimental work focused on the full analysis of chromatic dispersion evolution versus fibre stretching.

The delay line is tested in a fibre Mach-Zehnder interferometer. The experimental work is carried out using a spectral analysis of the interferometric mixing. A linear variation of the second order chromatic differential dispersion is observed versus high stretching. The stretched fibre dispersion evolution is scaled by comparison with unstretched fibre samples generating an equivalent group delay.
\end{abstract}

\section{KEYWORDS:}

Optical fibre, chromatic dispersion characterisation, broadband interferometry.

(1) Teacher Researcher, Ecole Militaire Polytechnique, BP 17 Bordj El Bahri, GGA 35320 Boumerdes Algérie.

(2) Professor, Equipe optique, IRCOM (UMR 6615) 123 rue A. Thomas, 87060

Limoges Cedex France 


\section{INTRODUCTION:}

In interferometric applications, classical delay lines, using mirror trains, have reached such levels of complexity $[1,2]$ that a cheaper and a simpler alternative would be very attractive. Using silica fibre ability to induce large and variable group delay when they are stretched [3], we develop an original solution proposing the replacement of classical delay lines by optical fibre ones [4].

The first phase of the implementation of a large stroke optical fibre delay line was focused on the improvement of the stroke to be reached with such devices. The experimental results have demonstrated that an air delay equal to two meters can be reproducibly generated by stretching a 100 meters long standard silica single mode wave-guide [5]. The main drawback was resulting from the variable coupled effects of dispersion and polarisation, leading to disturbed interferograms and low levels of fringe contrasts.

The second phase of the study investigated the polarisation control of a propagating optical field over the stretching process. The standard silica fibres were replaced by polarisation maintaining fibres. The experimental work was achieved in the $1.3 \mu \mathrm{m}$ spectral domain in order to minimise the chromatic dispersion effects. The results have demonstrated that the replacing of standard silica fibre by highly birefringent fibre has allowed the preservation of the polarisation coherence for the interferometric mixing [6].

This paper deals with the third phase of the experimental study. It consists in the accurate quantitative analysis of the chromatic dispersion evolution over very large stretching.

The study is performed in the frame of a coherent optic application by means of a Mach-Zehnder interferometer implemented with highly birefringent fibre. The instrument includes two optical fibre delay lines and a free beam air delay line. The main experimental work is carried out using a spectral analysis of the interferometric mixing denoted the channelled spectrum method [7].

After some theoretical considerations, we present experimental results, firstly devoted to characterise the implemented optical fibre delay lines, secondly to analyse the dispersion evolution versus fibre stretching.

\section{THEORETICAL CONSIDERATION:}

The dispersion analysis is achieved by means of an interferometric method.

In a two-beam fibre interferometer including an air delay line (Fig 1), the interferometric mixing spectrum can be expressed by:

$$
\mathrm{B}(v)=\mathrm{B}_{0}(v) \cdot[1+\mathrm{C}(v) \cos (\Phi(v))]
$$

Where $\mathrm{B}(v)=\mathrm{B}_{1}(v)+\mathrm{B}_{2}(v)$ is the total spectral power density as a function of the frequency " $v$ ", $\mathrm{B}_{1}(v)$ and $\mathrm{B}_{2}(v)$ are respectively the power spectral densities at the output of the two interferometric arms and $\mathrm{C}(v)$ expresses the contrast as a function of the frequency resulting from the spectral photometric unbalance between the two interferometric arms:

$$
\mathrm{C}(v)=2 \cdot \frac{\sqrt{\mathrm{B}_{1}(v) \cdot \mathrm{B}_{2}(v)}}{\mathrm{B}_{1}(v)+\mathrm{B}_{2}(v)}
$$


The phase shift " $\Phi(v)$ "can be decomposed as " $\Phi(v)=\Phi_{\mathrm{f}}(v)+\Phi_{0}(v)$ " where $\Phi_{\mathrm{f}}(v)$ is the phase shift introduced by the propagation in the fibres and $\Phi_{0}(v)$ results from the air path propagation.

The fibre contribution can be expressed by:

$$
\Phi_{\mathrm{f}}(v)=\beta_{1} \mathrm{~L}_{1}-\beta_{2} \mathrm{~L}_{2}
$$

where $\beta_{k}=n_{e k} \cdot \frac{2 \pi v}{c}$. ( $k=1$ or 2$)$ is the constant propagation, and " $L_{1}, L_{2}$ " are the fibre length in the two interferometric arms. It can be developed around a mean frequency $v_{0}$ :

$$
\Phi_{f}(v)=\sum_{i=0}^{n}\left(\frac{\partial^{i} \beta_{1}}{\partial v^{i}} \cdot L_{1}-\frac{\partial^{i} \beta_{2}}{\partial v^{i}} \cdot L_{2}\right) \cdot \frac{\left(v-v_{0}\right)^{i}}{i !}
$$

The air contribution is given by:

$$
\Phi_{0}(v)=\frac{2 \pi \cdot v}{\mathrm{c}} \cdot \Delta \mathrm{L}=\frac{2 \pi \cdot v_{0}}{\mathrm{c}} \cdot \Delta \mathrm{L}+\frac{2 \pi}{\mathrm{c}} \cdot \Delta \mathrm{L} \cdot\left(v-v_{0}\right)
$$

where " $\Delta \mathrm{L}$ " is the air path difference between the two interferometric arms.

Assuming that in this spectral range the terms higher than two can be neglected, the second order is fully sufficient to fit accurately the experimental analysis $[8,9,10]$. Thus, the total phase shift may be approximated by the sum of a linear term and a quadratic one:

*The linear term versus frequency " $v$ ", is the sum of the linear terms of equations (4) and (5). It represents the group delay between the two interferometric arms and can be cancelled by adjusting " $\Delta \mathrm{L}$ " air path difference so that:

$$
\Delta \mathrm{L}_{\text {air }}=-\frac{\mathrm{c}}{2 \pi} \cdot\left[\frac{\delta \beta_{1}}{\delta v} \cdot \mathrm{L}_{1}-\frac{\delta \beta_{2}}{\delta v} \cdot \mathrm{L}_{2}\right]
$$

*The quadratic term (of equation 4) results from the group velocity dispersion. It causes the main observable effects of differential chromatic dispersion, as contrast degradation and broadening of the fringe envelope and it can be determined by means of a spectral analysis known as "the channelled spectrum method"[7].

\section{EXPERIMENTAL SET UP:}

The experimental set up is shown in Fig 1. The Mach-Zehnder interferometer is implemented with a York HB 600 polarisation maintaining fibre. The cut-off wavelength is $\lambda_{c}=0.532 \mu \mathrm{m}$ and the beat length is $L_{b}=1 \mathrm{~mm}$.

The characterisation of the generated group delay, the induced losses and the fringe contrast versus stretching is carried out by using a laser diode emitting a $\lambda=670 \mathrm{~nm}$ mean wavelength. The emission has a $\Delta \lambda=10 \mathrm{~nm}$ spectral bandwidth when driven under the laser threshold.

For chromatic dispersion analysis, a high pressure Xenon lamp is used as source and the study is performed over a [650-850 nm] spectral range for which silica chromatic dispersion effects are important.

The field amplitude division at the input of the interferometer and the flux recombination at the output are performed by two beamsplitters. Therefore, two interferometric signals are available at the output of the interferometer.

The light source is linearly polarised and injected by means of microscope objectives into the two interferometric arm inputs, the optical fields being aligned with the fast 
axes of the fibres. At the output of the interferometer, an analyser is aligned with the fed neutral axis of the fibres.

Each interferometric arm includes an optical fibre delay line. In order to generate the group delay modulation, a length of fifteen-meters of fibre are wound and glued on a rubber rim. A hydraulic process drives the rubber rim radial expansion so that the fibre is uniformly stretched. This configuration allows good sharing of the applied stresses.

An air delay line consisting of a variable air path by motion of a motorised translation is integrated in one of the interferometric arm in order to allow a temporal analysis of the interferometric signals and characterise the optical fibre delay lines.

The two interferometric mixing available at the output of the interferometer are launched in two monomode standard fibres in order to perform a single mode spatial filtering and avoid spatial influence on the fringe contrast. The first one is focused on a silicon photodiode. The second one is spectrally analysed by means of a prism spectroscope with a 1780 pixel linear CCD detector.

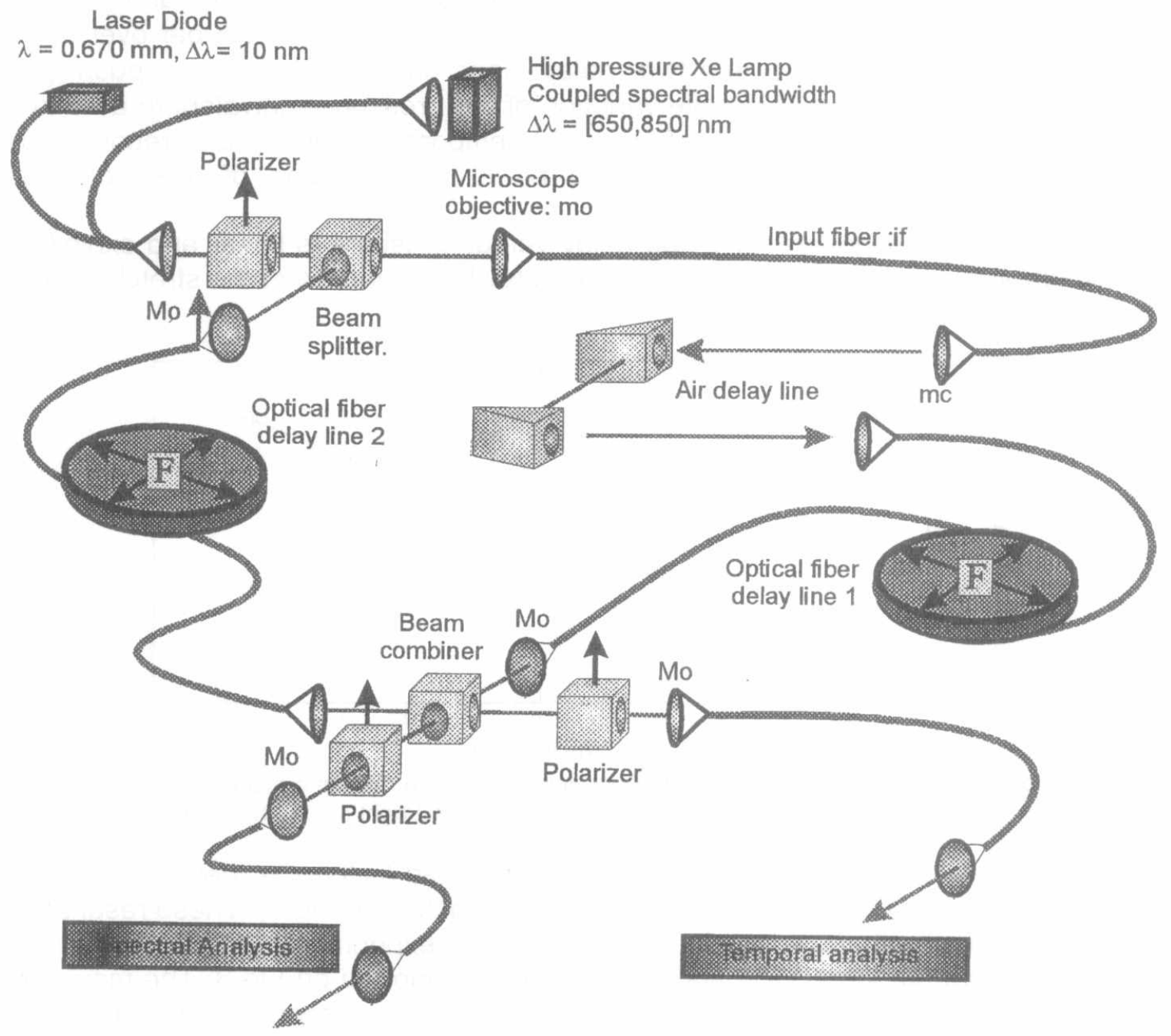

Fig 1: Experimental set up. 


\begin{tabular}{|l|l|}
\hline CS-7 & 5 \\
\hline
\end{tabular}

\section{EXPERIMENTAL RESULTS:}

Such a set up allows the achieving of both temporal and spectral analyses of the interferometric signals.

The temporal analysis is devoted to the confirmation of the results obtained in our previous experiments in the $1.3 \mu \mathrm{m}$ spectral range with standard silica fibre and polarisation maintaining fibre $[5,6]$.

The aim of the spectral analysis is to characterise the evolution of the differential second order chromatic dispersion.

\subsection{Temporal Analysis:}

The temporal analysis consists in the measurement versus fibre stretching of:

- The generated group delay.

- The losses induced on the stretched fibre during the lengthening process.

- The fringe contrast derived from the interferogram data.

The red laser diode, driven under the current threshold, is used as broadband source with a $\Delta \lambda=10 \mathrm{~nm}$ spectral bandwidth. An accurate equalisation of the group delay between the two interferometric arms is required for the observation of well contrasted fringes. The applied pressure is increased by step of 0.5 bar over a $0-6$ bar span successively in the two delay lines implemented in a push pull configuration. It results in a relative variation of the pressure equivalent to $a-6$ to 6 bar span. For each pressure, the fringes are temporally scanned and recorded by using an optical path variation induced by a linear motion of the motorised translation.

The variation of the generated group delay versus pressure is linear and exhibits a maximum air delay equal to $32 \mathrm{~cm}$ for each delay line unit ( $2 \%$ of the stretched fibre geometrical length, Fig 2).

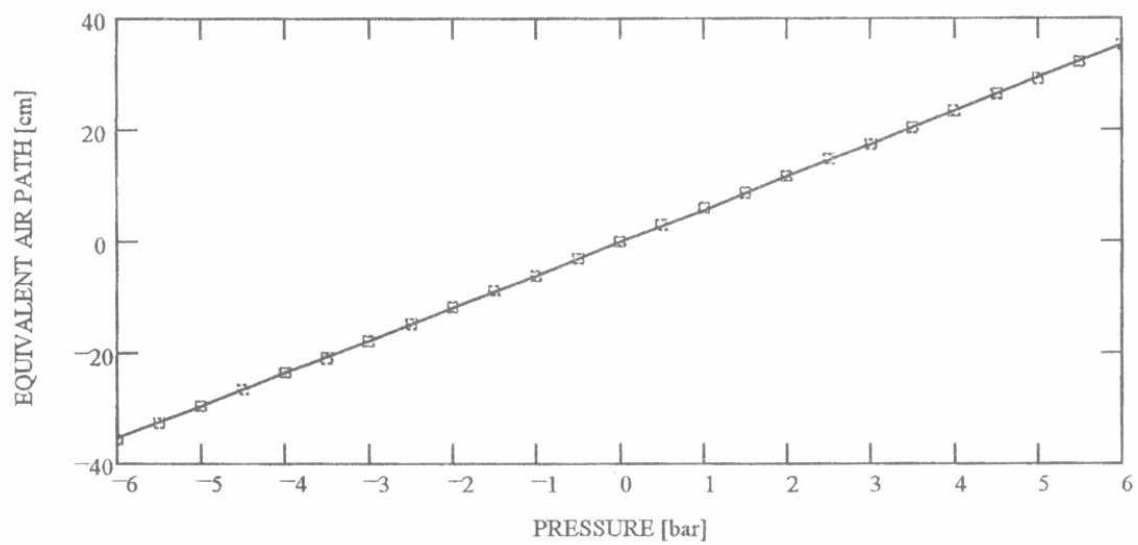

Fig 2: Global generated equivalent air path $(\mathrm{cm})$ versus pressure (bar) in a push pull configuration. Delay line number 1 left side ( 6 to 0 bar), number 2 right side ( 0 to 6 bar).

The induced losses are very low and can't be detected as long as they remain lower than the intensity noise to signal ratio typically equal to $1 \%$ (Fig 3 ). These results are in full agreement with those obtained in our previous experiments $[4,5,6]$

The fringe contrast versus the applied pressure is reported on Fig 3. The maximum of the curve $(97 \%)$ corresponds to the cancelling of the second order term of the phase shift. The noticed $50 \%$ contrast degradation is due to the additional dispersion introduced by the fibre stretching. On each side of the maximum, the unbalance of second order chromatic dispersion leads to fringe contrast decay. 


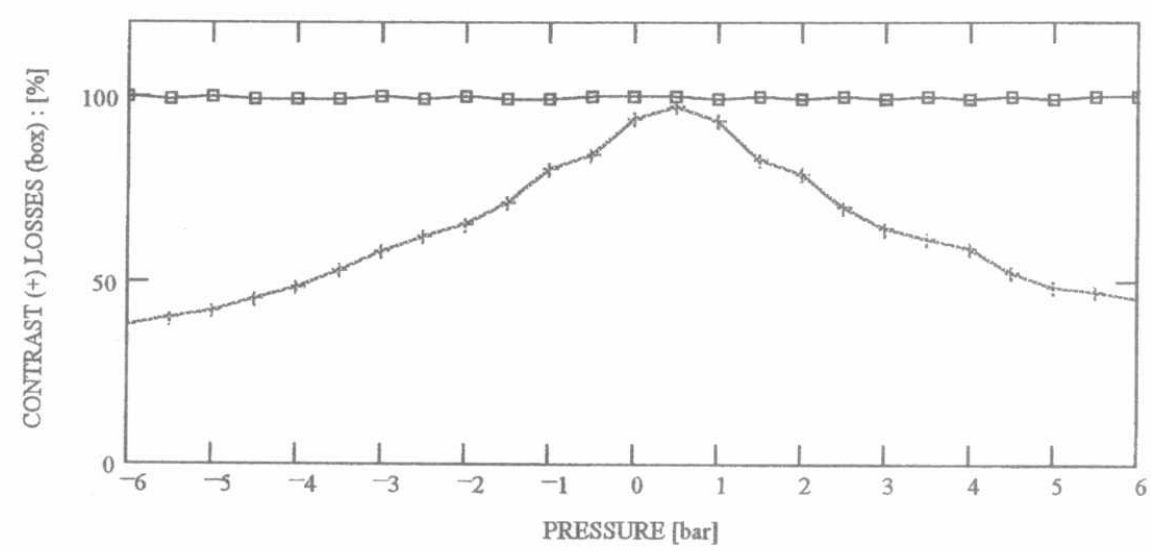

Fig 3: Fringe contrast $(+)$ and induced losses (box) versus pressure (bar). Delay line number 1 left side ( 6 to 0 bar), number 2 right side ( 0 to 6 bar).

\subsection{Spectral Analysis:}

This section deals with:

- The quantitative characterisation of the phase quadratic term evolution versus fibre stretching.

- The comparison between a stretched fibre dispersion evolution and an unstretched fibre one.

At the input of the interferometer, the Xe lamp replaces the laser diode.

In order to characterize and scale the chromatic dispersion variation versus fiber stretching two experimental configurations are compared (Fig 4).

In the first case, (denoted experiment A), the phase variation results from fiber stretching: The pressure, applied to the rubber rim, is increased by step of 0.5 bar over a 0-6 bar span. At each step, the zero group delay is adjusted using the air delay line. The resulting channeled spectrum is displayed on the linear CCD detector and the parabolic phase shift is determined as a function of the frequency.

In the second case (experiment $\mathrm{B}$ ), the phase shift varies versus the fiber length: The fiber length of the interferometric arm including the free beam air delay line is modified by replacing the input fiber labeled "if" (Fig 1). For each replacement, the zero group delay is adjusted and the associated phase shift determined.

Fig 4-a presents the spectrum of the high pressure Xenon lamp as seen at the interferometer out put. Fig 4-b reports a channeled spectrum recorded in the frame of experiment $\mathrm{A}$ for an applied pressure of 3 bar. Fig 4-c shows a channeled spectrum recorded in the frame of experiment B for a $56.5 \mathrm{~cm}$ length of the "if" input fiber (Fig 1). The central channel width variations point out the differential dispersion variation between the two recording.

Fig 4-d and 4-e present the parabolic phase shift versus frequency evolution for the two configurations. The different curves are derived from channeled spectrums recorded at different stretching states (experiment A) or for different lengths of the input fiber (experiment B). The experimental data are plotted and fitted with a third order polynomial regression. The fit uses a $v_{0}=750 \mathrm{~nm}$ mean frequency. A weak curvature exhibits a homogenous behavior of the different spectral source components. On the contrary an important curvature corresponds to a large variety of interference states. 

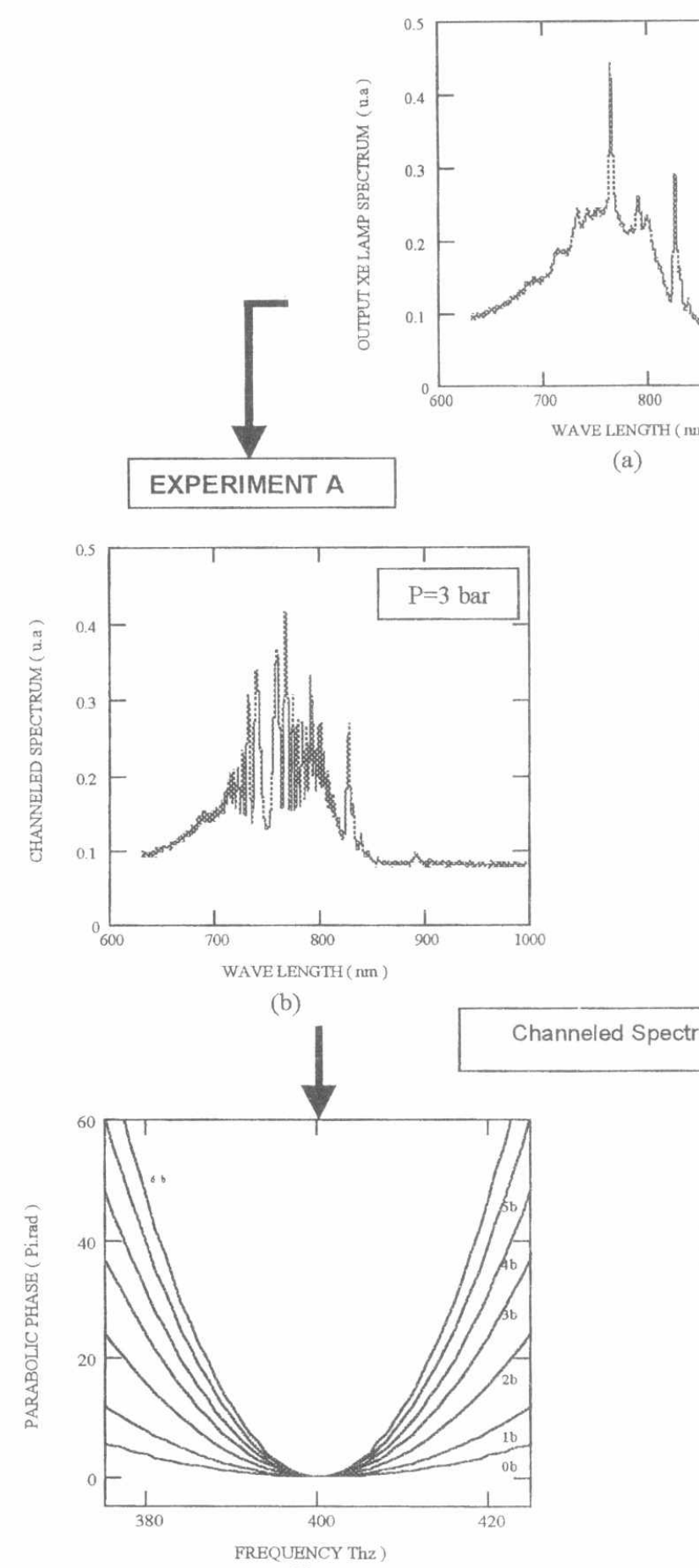

(d)

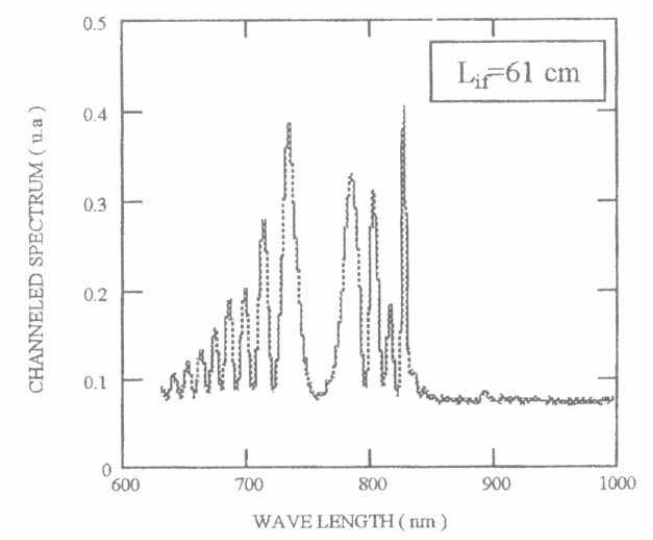

(c)

hanneled Spectrum Method

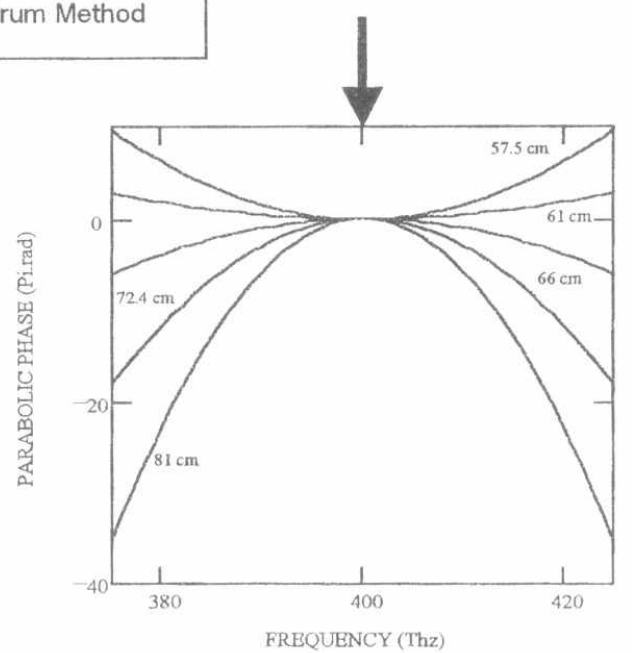

(e)

Fig 4

(a) Xe lamp spectrum as seen at the interferometer output.

(b) Channeled spectrum recorded at a pressure of 3 bar.

(c) Channeled spectrum recorded for a length of $61 \mathrm{~cm}$ of the input fiber "if".

(d) Evolution of the parabolic phase shift versus frequency for a [0 to 6] bar pressure variation.

(e) Evolution of the parabolic phase shift versus frequency for different lengths of the input fiber "if" $(57.5,61,66,72.4$ and $81 \mathrm{~cm})$. 
Fig 5 reports the second order chromatic dispersions variation of the two configurations versus the generated air path. The two curves exhibit a linear behavior of the second order chromatic dispersion coefficient. The slope is $a=1.03$ rad. $\mathrm{ps}^{2} \mathrm{~m}^{-1}$ for experiment $A$, and $\mathrm{a}=1,3$ rad. $\mathrm{ps}^{2} \mathrm{~m}^{-1}$ for experiment $\mathrm{B}$. Thus, in this spectral range, the chromatic dispersion of the stretched fiber remains smaller than the one of an unstretched fiber.

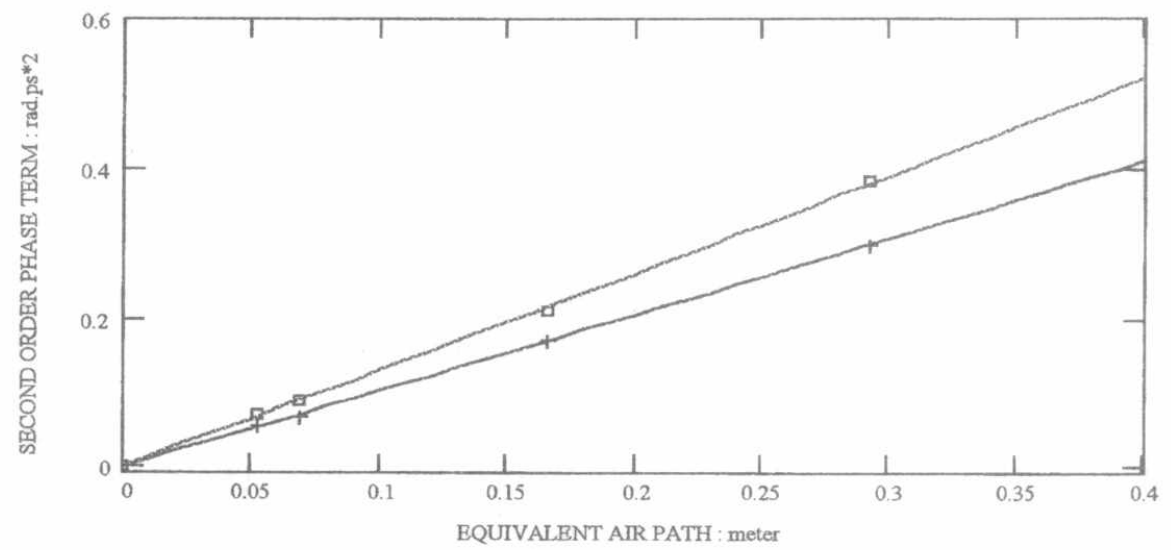

Fig 5: Experimental evolution of the second order phase term $\left(\mathrm{ps}^{2}\right)$ versus the generated air path (m). Stretched fiber (+) unstretched one (box).

The linear behavior of the parabolic phase shift variation versus stretching allows a simple and accurate calibration of the dispersion effects in the fiber interferometer.

\section{CONCLUSION:}

Chromatic dispersion evolution versus fibre stretching has been fully investigated in the frame of an interferometric application.

Both spectral and temporal analyses of the interferometric mixing have been achieved in a fibre Mach-Zehnder interferometer.

The temporal analysis has strengthened the fact that fibre stretching use for large delay generation is an interesting alternative solution for the replacements of classical delay lines and confirmed the results obtained in our previous experiments. The main result of the spectral analysis demonstrates that over the stretching, the parabolic phase shift variation is linear, allowing a simple and accurate calibration of the dispersion effects in the interferometer. With hindsight, the calibration of the dispersion effects in our interferometer, the polarisation control all over the operating spectral bandwidth and the photometry monitoring allow us to correct the fringe visibility decay as it is suggested in [11].

The achieved comparative study scales the extent of the additional dispersion introduced by the wave guide stretching. It demonstrates that the additional chromatic dispersion of a stretched fibre stays in an acceptable proportion and remains smaller than the one of an unstretched fibre generating the same group delay.

Future development will deal with the servo control of the optical path modulation. Two types of servo should be implemented; the first for the corrections of weak amplitude phase defects due to environmental perturbation $[12,13]$. The aim of the 
amplitude phase defects due to environmental perturbation [12,13]. The aim of the second is to allow the accurate monitoring over large stroke of the optical path generation.

On the other hand, we consider the replacing of classical systems of recombination currently used in our interferometers by integrated optics couplers. The goal is to achieve an all guided device in the frame of stellar interferometry applications devoted to onboard satellite instrument.

\section{REFERENCES:}

[1]. Faucherre. M., "Methods for optical pathlength compensation", ESO Conference on High Resolution Imaging by Interferometry, (part II), Ed.Merkle, 1991, pp.11791183

[2]. Booth A.J., Gilliand Y.A, Davis J, Tango W.J, Owens M.S., and Brown N, "Susi's path compensator system", ESO Conference on High Resolution Imaging by Interferometry, (part II), 1991, Ed.Merkle, pp.1127-1132

[3] Jacson D. A, Priest R., Dandridge A. and. Tventen A. B, "Elimination of drift in a single mode optical fiber interferometer using a piezoelectrically stretched coiler fiber" Applied Optics, Vol 19, n 19, p2926, 1980.

[4].Simohamed L.M, Delage L., Reynaud F., "An optical fibre delay line with a 318 mm stroke", Pure and Applied optics, Vol 5, p1005, 1996

[5]. Simohamed L.M., Reynaud F., "A two meter stroke optical fibre delay line ", Pure and Applied optics, Vol 6, p37, 1997.

[6] Delage L., Simohamed L.M., Reynaud F., "Utilisation de fibres optiques dans un interféromètre stellaire: démonstration en laboratoire des possibilités d'imagerie, étude et conception de lignes à retard métrique. ", Proceeding of ICSO'97, CNES/Toulouse, Décembre 97.

[7] Hen Tai Shang, "Chromatic dispersion measurement by white-light interferometry on metre-length single-mode optical fibres", Electron. Letter, 1981, 17, ( $\left.n^{\circ} 17\right), p$ 603605.

[8] Flavin D A, McBride R Jones J.D.C, Burnett J.G and Greenaway A H "Combined Temperature and strain measurement with a dispersive optical fibre Fourier transform spectrometer" Optics Letter, p 2167, V19, N24, 1994.

[9] Reynaud F., Alleman J.J., Connes P., "Interferometric control of fiber lengths for a coherent telescope array", Applied Optics, Vol. 31, N 19, p 3736-3743, 1992.

[10] Lagorceix H., "Application des fibres optiques unimodales à l'interférométrie stellaire", Thesis of Limoges University, 07-1995.

[11] Simohamed L.M., "Lignes à retard métriques utilisant l'étirement mécanique des fibres optiques en silice" Thesis of Limoges University, 01-1999.

[12] Burnett J.G., Grenaway A.H., McBride R., and Jones J.D.C, "Balancing optical path lengths in broadband fibre interferometers" Applied Optics V34 N 13, p2194, 1995.

[13] Connes P. and Reynaud F., "Fiber tests on a radiotelescope", Proceedings of the ESO Conference "High Resolution imaging by interferometry ", Ed Merkle and Beckers, 1117, 1988. 